\title{
Beauty trends, technology and cosmetic treatments in 20 I8-an Indian perspective
}

Keywords: fat freezing, pain of waxing, HIFU treatment, skin care products, dusky

\section{Introduction}

Many people consider the start of the New Year as an opportunity for self-improvement and nothing truly feels better than looking your best. Be it removing the stubborn pad of fat that has lasted forever, volumizing your smile, or saying goodbye to the pain of waxing, a new year holds immense potential to rejuvenate your mind, body and soul and we are here to make sure that you don't lose your way.

\section{What did we leave behind in 20|7}

Before we move forward we should learn from the year gone by 2017 sustained the tremendous growth being observed in the wellness industry with the launch of several new products and technologies helping to strengthen the industry.

\section{Cryolipolysis}

Cryolipolysis or 'fat freezing' was 2017's fastest-growing trend. Technologies like Cool sculpt and Cooltech helped numerous people in permanently removing pockets of fat like tummy bulge, bra fat pad, thighs and double chin, making them feels fitter.

\section{Neuromodulators}

Initially practiced by celebrities, Botox, Xeomin, etc. made further headway into the lives of the common Indians, being extensively used to decrease forehead and periocular fine lines. 2017 was the year of launch of German neuromodulator (like Botox) called Xeomin in India, which was an eagerly awaited molecule and 2018 is yet to see the effect of this molecule in the industry.

\section{The desire to be fair}

Here to stay, with IV glutathione making further inroads in tier II and III cities. Other skin lightening medications, lasers and peels also continued to be in demand and will be more successful in the future. A fair proportion of the population also desired a uniformly even skin tone irrespective of their shade. The current trend of the west, the "dusky" or the "tanned" look has still not caught up in our country.

Radiofrequency technology seem to have taken over the place of ablative $\mathrm{Co}_{2}$ lasers due lesser pain and reduced downtimes in treatment of acne scars, stretch marks, etc. Looks like MNRF (micro needle Radiofrequency) will be the gold standard for resurfacing in darker skin types due to increased safety and low downtime.

\section{Anti pollution skin care}

Various cosmetic companies have launched skin care range with high doses of antioxidants and polyphenols due to increasing pollution and smog. 2018 will tell us how much it is effective.
Volume 2 Issue 2 - 2018

\author{
Apratim Goel \\ Dermatologist \& Laser Surgeon, Cutis Skin Solution, India \\ Correspondence: Apratim Goel MD, Dermatologist and Laser \\ Surgeon, Cutis Skin Studio, Mumbai, India, \\ Email mdcutis@hotmail.com \\ Received: January 30, 2018 | Published: March 27, 2018
}

\section{What to look forward to in 2018 !}

\section{Technology}

2018 will likely continue the growth of the cosmetic industry with more people opting for holistic skin treatments. Newer technologies would help convert a lot of skeptics due to minimization of procedural side effects like pain, dyschromias and downtime.

\section{HIFU or high intensity focused ultrasound}

Better machines will provide sustained non-surgical facelifts over single session with almost no down time. With many more providers in the market, HIFU treatment seems to be emerging as the best technology for 2018.

\section{Body contouring devices}

"Face is the new body" seems to be coming true for 2018. There is a paradigm shift in focus below the neck to body. Cool tech, cool sculpt or newer entries like Sculpsure and Vanquish will continue to see increasing demand due to more people desiring toned physiques.

\section{Pico second lasers}

The possibility of delivering higher energy while protecting normal skin cells will further improve the treatment of pigmentation and freckles, whilst simultaneously leading to wholesome rejuvenation. However considering the cost of the technology and the benefit that pico second lasers have the existing technologies, this seems doubtful yet. India is a growing yet cost sensitive market and looks like pico second lasers will have to wait.

\section{Hair Reduction lasers}

They have continued to dominate the market for quite some years now and this will continue due to advances like better cooling attachments which lower the chances of side effects and cause minimal patient discomfort. Use of multiple wavelengths in one laser will definitely be the choice of the year. The triple wavelength Primelase laser and Soprano Ice seems to be the hot sellers. 


\section{Hair loss solutions}

There has been an explosion in the newer treatments and technologies for hair loss which explains the increasing demand for hair loss solutions. Use of Korean threads in scalp, hair stem cells and robotic hair transplant surgeries have already taken a leap in 2017 and we expect it to peak on 2018-19.

\section{Trends}

For those who have their hearts set on looking better, non-surgical treatments will continue to be more popular than going under the knife.

\section{Fillers}

Thanks to celebrities such as Kylie Jenner, treatments such as lip augmentation are likely to top 2018's popularity list along with fillers on other parts of the face for compensating volume loss. Fillers have evolved beyond the original hyaluronic acid to others that bring greater specificity and nuance, so that skilled injectors would be able to further optimize results. Voluma, Volbella, Boletero, Cellifique (debuted in Europe, 2017), etc. have only added to our armamentarium. We are finally becoming artists with a full palette of paints.

Microtreatments are including micro-blading/pigmentation for semi-permanent eyebrow enhancement, lip colour enhancement and scalp tattooing to give an impression of fullness, as also microneedling treatments for improving pores, wrinkles, scars and sun damage will continue to gain momentum in 2018.

Teledermatology may grow because of its convenience. Especially for common skin problems like acne where patients could click and send a few pictures to the doctor, answer a few Q's about their skin and a personalized acne treatment protocol would then ship right at their door.

Mesolipolysis using deoxycholic acid (kybella/geolysis/fatX), an injectable which helps in reducing moderate fat areas especially double chin has become the latest "in" thing and should make it's way in the next year.

Vaginal tightening devices like Thermi Va, Fiore and Femilift have taken the West by storm, however the Indian market hasn't been in acceptance of this trend. However, 2018 could see an emergence of these technologies in India, helping women feel rejuvenated.

\section{Make up}

Finally, dermatologically tested brands with natural ingredients will be increasingly in vogue and will present a growth opportunity for companies that operate in this high volume market. Air brush technique will be the flavor of the year.

\section{Skin care products, ingredients and more}

\section{Medical facials}

Instead of simply using scented soaps and applying traditional facials, medical facials use prescription strength ingredients like Retin-A, Vitamin $\mathrm{C}$, and Alpha and Beta Hydroxy acids thus providing much better efficacy.
These facials combined with laser technologies will continue their upward trajectory. The constant exposure to pollution will further propagate anti-pollution medifacials. These consist of lymphatic massage to drain toxins and use of deep sea products that help in detoxification and clearing of skin.

Thanks to insta trends like \#Sunday facials face masks are here to stay and be more popular than exotic ingredients like charcoal, wine, caviar, snail secretions, etc.

People will continue to spend extra on premium skin care products like those of ZO skin health, FCL, fillerina and supplements like celergan, lycowhite, crystal tomato and CoQ10.

\section{Treatments and customer demand}

Men treatments: In the current era, a gender neutral cosmetic industry that once catered mainly to women is now seeing tremendous growth in the male populace. The most opted for treatment among men being for hair loss. Correcting imperfections such as dark circles, giving an outline to the beard and eyebrows have become habitual actions for the consumer, who seeks androgynous gestures for a result of a gentleman's style. Men skin and hair care products are likely to be in high demand.

Body contouring in women: On the other hand women seem to be interested the most in anti-aging and body contouring treatments. 'Body is the new face' seems to be the new mantra. Body polishing and care of neglected parts like underarms, neck, hands, and feet would be the focus. New technologies like Thermi and Miradry which promise an end to sweaty armpits in a couple of sittings will continue to accelerate the trend of caring for your body.

Ozone therapy: Ozone therapy is taking the beauty market by storm. Antibacterial, antifungal and antiviral, a drip of ozone will invigorate you and also make your skin glow. It will increase oxygen binding in body and hence oxygen perfusion of skin and other organs.

IV infusion therapy: Intravenous delivery of drugs is as old as mankind. Then what's new? The new trend is delivery of a cocktail of vitamins, hormones, nutrients and minerals intravenously into the blood stream. This not only ensures delivery of right amount of ingredient into the blood but also fastest mode of deliver. Used in cosmetic industry for skin lightening, hair loss, sensitive skin, jet lag etc.

\section{Conclusion}

2018 seems to be another promising year with loads of goodies in our kitty. I have my fingers crossed to see in which direction the wind blows. Super excited about the thought of being able to work with newer trends and technologies.

\section{Acknowledgements}

None.

\section{Conflicts of interest}

The authors declared that there are no conflicts of interest. 